\title{
The Role of the insula in Internet Gaming Disorder
}

\author{
Ofir Turel ${ }^{1,2^{*}, * *}$, Qinghua $\mathrm{He}^{3,4,5,6,7, *, * *}$, Lei Wei ${ }^{3}$, Antoine Bechara ${ }^{2}$ \\ ${ }^{1}$ Information Systems and Decision Sciences, California State University, Fullerton, CA, \\ United States \\ ${ }^{2}$ Brain and Creativity Institute, University of Southern California, Los Angeles, USA \\ ${ }^{3}$ Faculty of Psychology, Southwest University, Chongqing, China \\ ${ }^{4}$ Key Laboratory of Cognition and Personality, Ministry of Education, Southwest University, \\ Chongqing, China \\ ${ }^{5}$ Chongqing Collaborative Innovation Center for Brain Science, Chongqing, China \\ ${ }^{6}$ Southwest University Branch, Collaborative Innovation Center of Assessment toward Basic \\ Education Quality at Beijing Normal University, Chongqing, China \\ ${ }^{7}$ Institute of Psychology, Key Laboratory of Mental Health, Chinese Academy of Sciences, \\ Beijing, China
}

* These authors have made equal contributions.

** Corresponding authors:
(a) Ofir Turel, 800 N. State College Blvd., Fullerton CA, USA 92834, Email: oturel@fullerton.edu; Phone: +1 (657) 278-5613
(b) Qinghua He, Faculty of Psychology, Southwest University, Chongqing, China, email: heqinghua@gmail.com; Phone: +(86)-136-4769-1390

Funding: QH was supported by research grants from the National Natural Science Foundation of China (31972906), Open Research Fund of the Key Laboratory of Mental Health, Institute of Psychology, Chinese Academy of Sciences (KLMH2019K05), and the High-end Foreign Expert Introduction Program (G20190022029).

\begin{abstract}
Internet Gaming Disorder (IGD) is a concerning issue that requires further research. Here, we seek to examine its neural etiology with an emphasis on the role of the insula. To do so, we relied on the tripartite neurocognitive model of addictive behaviors as applied to IGD. We hypothesized that (1) video game cues will elicit stronger reward system activation, and weaker prefrontal activation in gamers vs. controls, (2) the IGD scores of gamers will be positively associated with activation of the reward system and negatively with activation of prefrontal regions, (3) deprivation from video gaming will result in increased activation of the insula, when gamers are exposed to videogame cues vs. to neutral cues, and (4) in deprivation conditions, there will be positive and negative coupling, respectively, between activation of
\end{abstract}

This is the author manuscript accepted for publication and has undergone full peer review but has not been through the copyediting, typesetting, pagination and proofreading process, which may lead to differences between this version and the Version of Record. Please cite this article as doi: $10.1111 / \mathrm{adb} .12894$ 
the insula and the reward and prefrontal regions in gamers. We tested these hypotheses with a design with one between-subjects factor (gamers vs. controls), and two within-subjects factors: stimuli (gaming vs. neutral; for all participants) and session (deprivation vs. satiety; only for gamers). Findings based on functional magnetic resonance imaging (fMRI; applied to all 52 subjects, 26 gamers and 26 controls) and psychophysiological interaction (PPI; applied to the 26 gamers) engaged in a video-reactivity task supported our assertions. The IGD score positively correlated with activity in the right ventral striatum and negatively with activity in the right dorsolateral prefrontal cortex (DLPFC). Left insular cortex activity was the highest when observing video gaming cues under deprivation. Lastly, there was an increased coupling between the left insula and left ventral striatum and a decreased coupling with left DLPFC when observing video gaming cues compared to when watching control videos in the deprivation condition.

Keywords: insular cortex, Internet Gaming Disorder, Tripartite Neurocognitive Model, Decision Making, Interoceptive awareness, fMRI

\section{Introduction}

Video gaming has become a popular form of leisure activity. For example, in China, there were 554 million mobile game users in 2017, out of which 7.5\% played for over 2 hours per

day ${ }^{1}$. While low to moderate levels of gaming may induce some beneficial brain plasticity ${ }^{2}$, improved hand-eye coordination ${ }^{3}$, and reduced interest in illicit substances ${ }^{4}$, high-levels of gaming can sometimes be excessive and adversely affect gamers' normal functioning by replacing other desirable activities ${ }^{5,6}$.

Thus, excessive gaming can manifest in symptoms that are consistent with those observed in behavioral addictions (i.e., salience [video gaming dominates people’s thoughts and behaviors], tolerance [there is an increased need to video game more], mood modification [video gaming creates relief], conflict [video gaming conflicts with desirable behaviors], withdrawal [deprivation creates dysphoria], and relapse [attempts to quit video gaming are 
doomed to fail] $)^{7,8}$. While the recent Diagnostic and Statistical Manual of Mental Disorders (DSM-5) does not yet formally recognize this phenomenon as a mental disorder, it calls it Internet Gaming Disorder (IGD) and advocates for more research on it ${ }^{9}$. Similarly, the World Health Organization calls it Gaming Disorder in its $11^{\text {th }}$ Revision of the International Classification of Diseases (ICD-11) ${ }^{10}$. It defines it as a pattern of video-gaming that is characterized by increased salience of gaming, loss of control over gaming, and continuation of or increase in gaming despite its negative consequences. It is vital to study IGD given its potential adverse impacts on users, their families, and possibly nations ${ }^{11}$. Hence, we seek here to understand better the etiology of IGD, and specifically its neural underpinnings.

Because addictive behaviors can be viewed as a form of maladaptive responses to relevant cues ${ }^{12,13}$, it has been argued that IGD can be explained with a Tripartite Neurocognitive Model ${ }^{14}$. This model is based on the extension of traditional dual-process models of maladaptive decision making ${ }^{15,16}$ that also accounts for interoceptive awareness processes. Ineroceptive awareness processes encompass the translation of somatic signals (e.g., in states of deprivation) into a subjective state of desire ${ }^{17}$. The dual-process part of this model suggests that the development and maintenance of IGD relate to hyperactivity of the reward (amygdala-ventral striatal dependent) system in response to videogame cues, coupled with the weakening of inhibition abilities (prefrontal cortex, and specifically DLPFC dependent; it can also include the ventromedial prefrontal, orbital frontal and anterior cingulate cortices) in response to videogame cues. The third system that extends this model is the interoceptive awareness system (insular cortex [insula] dependent), which senses somatic signals of deprivation from videogame reward, and translates them into a subjective desire. This, in turn, exerts an influence on the balance between the reward- and inhibition- systems. Specifically, engaging the insula hijacks or weakens prefrontal activity (negative coupling), and 
exacerbates or enhances dopaminergic circuits (positive coupling) ${ }^{18-20}$. When interoceptive signal processing is disrupted, for example, through lesions to the insula, the desire to seek reward resulting from deprivation is reduced, and individuals can more easily extinguish addictive behaviors ${ }^{21}$.

This model has been shown to explain several addictive behaviors ${ }^{22,23}$. In addition, the roles of the reward and inhibition systems have been demonstrated in prior IGD studies ${ }^{24,25}$. What is missing for a more comprehensive theoretical account of the brain underpinnings of IGD is a focus on the role of the insula, and an integrative examination of the Tripartite Neurocognitive Model. We address this gap in this study.

Specifically, systematic reviews provide preliminary but inconsistent support for the dualprocess assertions (but less so to the existence of the third system), by showing that IGD cases differ from controls in the activation of the abovementioned regions ${ }^{6}$. Nevertheless, past results were somewhat inconsistent. For example, Ko, Liu, Hsiao, Yen, Yang, Lin, Yen, Chen ${ }^{26}$ found increased activity in response to video gaming cues in the reward system but also in prefrontal regions in IGD cases; and Lee, Lee, Chun, Cho, Kim, Jung ${ }^{27}$ found compromised (reduced) frontal functioning in IGD cases. The inconsistencies may stem, in part, from the yet-to-be agreed-upon measures and cutoffs for classifying people as IGD cases ${ }^{28}$. In addition, there is limited evidence for the relevance of the interoceptive awareness system in IGD. To bridge these gaps, extend the dual system view by integrating it with activations of the interoceptive awareness system in response to cues under deprivation conditions. Here, we avoid the use of artificial cutoffs by treating IGD as a continuous concept that captures the level of addiction-like symptoms that video-gamers experience. 
Given that gaming drives dopamine release in video gamers ${ }^{29}$ and can become habituated and less-inhibited ${ }^{30}$, our first hypothesis (H1) is that video game cues elicit stronger reward system activation and weaker prefrontal activation in gamers vs. controls. Based on the dualprocess model of addiction ${ }^{12,15,16}$, and in line with evidence from other addictive behaviors ${ }^{15}$, our second hypothesis (H2) is that the IGD scores of gamers are (a) positively associated with activation of the reward system and (b) negatively associated with activation of prefrontal self-control regions. Based on the Tripartite Neurocognitive Model extension to IGD, we test the third hypothesis (H3) that deprivation from video gaming will result in increased activation of the insula when gamers are exposed to videogame cues vs. to neutral cues. The same model suggests that this activation increases reward system activity and decreases prefrontal activity through coupling. As such, our fourth hypothesis is that in deprivation conditions, there will be (H4a) positive coupling between the insula and the reward system and (H4b) negative coupling between the insula and prefrontal self-control regions. We test H1 through comparing fMRI responses to video gaming cues in gamers vs. controls, H2 through correlational analyses of fMRI responses and IGD scores, and H3 through comparing fMRI responses to neutral vs. video gaming cues in deprived gamers. Lastly, H4 is tested with PPI analysis applied to deprived gamers. It links the activation of the hemisphere of insula identified by testing $\mathrm{H} 3$ to prefrontal and reward system activations. This design allows testing between- and within-subject differences in response to video gaming stimuli and extending prior works that have primarily overlooked within-subjects differences between video gaming deprivation and satiety conditions.

\section{Methods}

\section{Participants}


Fifty-two healthy volunteers read and signed an informed consent form, which was approved by the Institutional Review Board of a research university. They were recruited through two types of flyers. One flyer targeted intensive League of Legends (LOL) gamers (advertised as playing about 3 hours of hours/day and having $>1.5$ years of LOL experience). The other recruited controls who do not play LOL and have not played videogames in the last six months. We focused on LOL (a multiplayer battle arena game) because it is highly popular; it had 100 million monthly active players in $2016^{31}$. We ultimately recruited 26 intensive LOL players (LOL or gamers, 6 females; $\mathrm{M}_{\text {age }}=20.46 \pm 2.10$ years), and 26 age- and sex-matched controls (CNT, 6 females; $\mathrm{M}_{\text {age }}=20.69 \pm 2.21$ years). The sample was male-dominant, which presumably represented the population of gamers in China ${ }^{1}$

\section{Procedures}

Participants came to the lab for one or two sessions; the choice depended on group affiliation (LOL or CNT). During their first session, both groups were interviewed regarding their LOL play patterns. The criteria for intensive LOL players were 1) having at least 1.5 years of LOL experience, and 2) spending about 3 hours per day playing LOL. The criteria for the control group were 1) having no experience with LOL, and 2) not playing videogames in the last six months. All participants were required to be right-handed. Both groups were next interviewed using a computerized version of the Structured Clinical Interview for DSM-5 (SCID-5) regarding current or lifetime neurological disorders that may affect the results. These included psychoses, major depressive episode, and major depressive disorder, heavy drinking, substance abuse, schizophrenia, current and history of anxiety disorders, and bipolar disorder. Participants were also screened for alcohol use using AUDIT (Alcohol Use Disorders

\footnotetext{
${ }^{1}$ In 2015, 73\% of gamers in China were males (see: https://www.go-globe.com/online-gaming-china/). The proportion in our sample was 20/26=76.9\%.
} 
Identification Test), and smoking dependence using FTND (Fagerström Test for Nicotine Dependence). There were no differences between the two groups in alcohol (gamers: $0.62 \pm$ 0.75 ; controls: $0.42 \pm 0.70 ; \mathrm{t}(50)=0.95, \mathrm{p}=0.35$ ) and cigarette use (gamers: $0.31 \pm 0.47$; controls: $0.54 \pm 0.51 ; \mathrm{t}(50)=-1.70, \mathrm{p}=0.10)$. Participants were also questioned regarding abnormal uncorrected vision. No exclusions were made based on any of the criteria; no current or lifetime disorders were reported.

Next, both groups reported age and sex. The gamers completed an online survey that included several descriptive variables related to LOL play (see details below). All participants were scanned in an fMRI device while performing a video reactivity task. Cue reactivity tasks are commonly used to elicit BOLD signals in brain regions that are relevant to addictive behaviors ${ }^{32}$. Gamers were invited to return for another fMRI scan, which was scheduled for at least one month after the first session (mean difference: $38.4 \pm 5.1$ days, 31-45 days). In the second scan, they finished the same fMRI tasks but under a different condition. Specifically, we employed two conditions, normal play and deprivation, the order of which was counterbalanced across participants. In one session, gamers were asked to avoid LOL playing from 6 PM of the previous day (i.e., deprivation condition). In the other session, they were asked to play normally. They were invited to play LOL right before the scan (i.e., satiety condition). The scan of the deprivation condition took place between 10-11 AM, to ensure exposure to the same deprivation duration. In all between-sessions analyses, results remained virtually identical when we added session order as a factor. We checked manipulation effectiveness by asking participants to rate their craving for playing LOL in both sessions, right before the scan. The control group was scanned only once, because it was naturally deprived of LOL, and had no basis for craving LOL.

\section{Survey}


The survey captured age and sex in both groups. It captured years of experience with videogames (not just LOL) and LOL daily use frequency only in the LOL group. It also captured IGD scores in the LOL group with the IGD-Short Form scale by Pontes, Griffiths ${ }^{33}$ on a 1-7 Likert Scale; see items in supplementary materials $\mathbf{A}$. The scale was reliable ( $\alpha=$ 0.877). It captures the last three months' extent of sensing DSM-5 addiction-like symptoms concerning playing LOL. We used a forward-backward translation process to ensure equivalency between the original English version and the translated Chinese version.

\section{Video Reactivity Task}

This task used videos instead of static images because LOL is a dynamic online game. We hence assumed that gaming videos would serve as more potent cues for gamers. In this task, the brain activity was measured when participants watched two categories of video stimuli: LOL videos and control videos (see example stimuli in supplementary materials B). There were 12 videos (six LOL and six control videos) in total. Each video was 30 seconds long. The LOL videos included screen captures of someone who played LOL. The control videos were selected from news reports and tried to match the movements, pace, and scenes in the LOL videos. In a pilot study, we asked 15 participants (not included in the main study) who had some LOL experience to rate their familiarity with, perceived vividness, and colorfulness of all videos, using a 1-7 Likert scale. Results suggested that the videos were matched on all three dimensions (see statistics in Table S1 of the supplementary materials $\mathbf{C}$ ). The task used a block design: each video played as one block, preceded by two seconds of fixation display “+," and followed by 20 seconds of a blank screen. The order of the presented videos was counterbalanced across participants (LCCLLCCLLCCL or CLLCCLLCCLLC²).

\section{fMRI Protocol and Analyses}

$\overline{{ }^{2} \mathrm{~L}=\mathrm{LOL} \text { video; } \text { and }=\text { control video }}$ 
FMRI protocol details are given in supplementary materials D. Data preprocessing and statistical analyses were performed with FSL (version 6.0.1, www.fmrib.ox.ac.uk/fsl). fMRI images were realigned to compensate for small residual head movements ${ }^{3}$. Data were spatially smoothed using a 5-mm full-width-half-maximum (FWHM) Gaussian kernel. The data were filtered using a nonlinear high pass filter with a 100-second cut-off. A two-step registration procedure was used whereby EPI images were first registered to the MPRAGE structural image, and then into standard MNI space, using affine transformations. Registration from MPRAGE structural image to standard space was further refined using FNIRT nonlinear registration. Statistical analyses were performed in the native image space, with the statistical maps normalized to the standard space prior to higher-level analyses. The data were modeled at the first level using a general linear model (GLM) within FSL's FILM module.

Brain activations for both LOL videos and control videos were modeled in the single-subject level. The boxcar response was convolved with the canonical hemodynamic response function (HRF, double-gamma) to generate regressors used in the GLM. Temporal derivatives were included as covariates of no interest to improve statistical sensitivity. Resting periods were not explicitly modeled, and therefore constituted an implicit baseline. The six movement parameters were also included as covariates in the model. Higher-level random-effects models were tested for group differences using FMRIB's Local Analysis of Mixed Effect stage 1 only with automatic outlier detection.

The activity difference between LOL and control videos were entered at the second level analysis. Two comparisons were made between groups/sessions. First, the LOL and control groups were compared to examine activity differences between the LOL videos and the control videos. Second, the activity difference was also compared between satiety and

\footnotetext{
${ }^{3}$ Translational movement parameters never exceeded 1 voxel in any direction for any participant.
} 
deprivation sessions in the LOL group. This was done to understand the modulatory effect of deprivation better. Group images were evaluated with a height threshold of $Z>3.1$ and a cluster probability of $\mathrm{p}<0.05$, corrected for whole-brain multiple comparisons based on Gaussian random field theory.

To better illustrate the brain activity differences in each condition and each group, regions of interest (ROI) were created from clusters of voxels with significant activation differences in the voxel-wise analyses. Analyses were performed by extracting parameter estimates (betas) of each type of stimuli from the fitted model and averaging across all voxels in the cluster for each participant. To restrict the number of multiple comparisons, we only extracted the following ROIs: right ventral striatum (Figure 2A, Figure 4B), left (Figure 4C), and right (Figure 2B, Figure 4D) DLPFC, and left insular cortex (Figure 4A). Family-wise errors were corrected using Bonferroni correction. For analysis of correlations between behavioral and ROI data, robust regression was used to minimize the impact of outliers in the behavioral data, using iteratively reweighted least-squares implemented in the robustfit command in MATLAB. Reported $r$-values reflect (non-robust) Pearson product-moment correlation values. In contrast, the reported $p$-values and regression lines are based on the robust regression results.

To better understand the connectivity between brain regions, psychophysiological interaction (PPI) analysis was performed using data from all sessions with LOL participants. A time series of significantly activated voxels in the left insular cortex cluster was extracted for each participant. The PPI analysis followed FSL community guidelines (https://fsl.fmrib.ox.ac.uk/fsl/fslwiki/PPI). For each individual session, we included LOL video, control video, insula time course, as well as two interactions of LOL video by insula, and control video by insula. Group analyses were performed to examine group differences 
between interactions and to specifically find whether brain connectivity with left insula differed between LOL and control videos. Group images were evaluated with a height threshold of $\mathrm{Z}>3.1$ and a cluster probability of $\mathrm{p}<0.05$, corrected for whole-brain multiple comparisons based on Gaussian random field theory.

\section{Results}

\section{Behavioral Results}

The two groups were matched on age $(t(50)=0.39, p=0.70)$ and sex. The LOL group had, on average $8.11 \pm 2.75$ (standard deviation) years of online video gaming experience. In the past three months, they spent, on average $3.88 \pm 1.45$ days per week on LOL. They spent about $2.42 \pm 0.95$ hours on each game. Their average IGD score was $3.90 \pm 1.13$ (2.63 to 6.63). The mean of 3.90, which is based on a sample of heavy gamers, is much higher than the mean reported by the original study of this scale, which was based on a sample of the general population of gamers (2.00 on a 1-5 Likert scale, which is equivalent to 2.93 on a 1-7 Likert scale). While there is no formal way to classify people as clinical IGD cases or not, our results increase confidence in our focus on people with a range of and some with relatively high IGD scores. Because the video task required only passive viewing, no behavioral measures were generated. Nevertheless, participants were monitored by an eye-tracking system to ensure they were awake and attentive during the video presentation. Right before the scan, the LOL group reported their craving on a 7-point Likert scale $(1=$ no craving at all, 7 = want to play really badly). Results pointed to significantly different cravings in the two sessions (deprivation: $6.27 \pm 0.60$; satiety: $5.23 \pm 0.71$; $t(25)=5.78, p<0.001$ ), suggesting that our manipulation produced the desired craving.

\section{fMRI Results}


First, a second-level random-effects model was estimated in order to examine activation differences between the groups on LOL-CNT video contrast. This was done with a mixed 2way ANOVA with stimuli as a within-subjects factor (LOL vs. control video) and group as a between-subjects factor (LOL vs. CNT group). Results suggested no significant differences in activation based on either stimuli or group (direct effects). However, stimuli x group interaction had significant effects on the following brain regions (Table $\mathbf{1}^{\mathbf{4}}$ ): right ventral striatum (Figure 1A), right DLPFC (Figure 1B), left frontal pole (Figure 1C), and bilateral ventromedial prefrontal cortex (VMPFC, Figure 1D). Results suggested that the right striatum had a significantly stronger activation when gamers watched LOL videos than when watching control videos; and when controls watched control videos compared to when watching LOL videos (Figure 2A). In contrast, when gamers watched LOL videos, they showed lower prefrontal activity than when watching control videos, while the control group did not show such differences (Figure 2B). Furthermore, using the LOL-CNT contrast, the IGD score significantly positively correlated with activity in the right striatum (Figure 2C), and negatively correlated with activity in the right DLPFC (Figure 2D).

***Insert_Table_1_,_Figure_1_and_Figure_2_About_Here***

Next, a second-level random-effects model was estimated to examine activation differences between the two sessions (deprivation vs. satiety) of the LOL group (i.e., gamers only). This was done with a within-subjects two-way ANOVA, in which "stimuli" and "session" were within-subjects factors. Results pointed to a significant main effect of "session" in the left (Table 2 and Figure 3A) and right insular cortex (Table 2 and Figure 3B). No voxels

\footnotetext{
${ }^{4}$ Currently there are no tools for calculating effect sizes for fMRI data. We therefore reported the effect sizes in all behavioral analyses, but not in fMRI analyses.
} 
showed main effect of stimuli. There were significant interaction effects in the right ventral striatum (Table 3 and Figure 3C), bilateral DLPFC region (Table 3 and Figure 3D), as well as the left insular cortex (Table 3 and Figure 3E). The activity in the left insular cortex was extremely higher when watching LOL videos in the deprivation condition than in all other conditions (Figure 4A). Activity in the right striatum was higher in deprivation condition than in satiety condition when participants watched LOL video (Figure 4B). Bilateral DLPFC activation was lower in the satiety condition than in the deprivation condition when watching LOL videos (Figure 4C, Figure 4D).

***Insert_Table_2_Table_3_and_Figure_3_and_Figure_4_About_Here***

Last, we used the left insula, which showed an interaction between session and stimuli, as a region of interest and applied PPI analysis. Results showed increased coupling between the left insula and left ventral striatum when watching LOL videos compared to when watching control videos in the deprivation condition, but not in the satiety condition (Table 4 and Figure 5). It also showed a decreased coupling between the left insula and left DLPFC when watching LOL videos compared to when watching control videos in the deprivation condition, but not in the satiety condition (Table 4 and Figure 5).

***Insert_Table_4_and_Figure_5_About_Here***

\section{Discussion}

This study sought to empirically test the Tripartite Neurocognitive Model of IGD, and more broadly, to provide evidence for the relevance of brain systems that were implicated in other addictive behaviors to the case of video gaming. More importantly, this study addressed the question of whether deprivation from external sensory cues, such as video games, has the capacity to induce a deprivation state similar to that induced by ingested substances, which 
generate interoceptive signals mediated through the insula. Because deprivation is needed for activation of the interoceptive process described in the tripartite model, we used a design with one between-subjects factor (gamers vs. controls), and two within-subjects factors: stimuli (LOL vs. neutral; for all participants) and session (deprivation vs. satiety; only for gamers). We focused on one popular and possibly “addictive” video game, namely League of Legends 34.

The first hypothesis focused on the general involvement of reward and self-control centers in the brain in responding to video gaming cues. Because video gaming is intrinsically rewarding ${ }^{29}$, we expected that video gaming cues drive stronger reward system activation, compared to neutral cues, especially in gamers. Because through repetition and learning gamers habituate their behavior, beyond awareness and self-control ${ }^{30}$, we expected that video gaming cues drive weaker prefrontal activation in gamers. Our results supported the first hypothesis and showed that the ventral striatum was more strongly activated and that the left frontal pole and DLPFC were more weakly activated in gamers in response to game cues than in controls. These results suggest that it is reasonable to posit that video gaming can overtime sensitize the brain in such a way that video gaming becomes increasingly automatic and dissociated from long-term motives ${ }^{12,13}$. Because this process is necessary for IGD development ${ }^{35,36}$, future research can rely on such findings and examine the processes through which normal video gaming transitions into IGD.

The second hypothesis addressed the dual-system perspective of addiction ${ }^{15,37}$. Predicated on the idea that IGD may be similar to other addictive behaviors ${ }^{38,39}$, we expected IGD scores of gamers to be positively associated with reward system activation and negatively with inhibition system activation. The observed positive associations between IGD scores and right ventral striatum activity and negative association with the right DLPFC activity supported our 
hypothesis. These results are in line with several studies on IGD ${ }^{6}$, but point to possible differences between excessive use of different technologies. While here we observed dysregulation deficits in both the reward and self-control brain systems, in the case of social media, excessive use scores manifested primarily from hyperactivity of the reward system, and the prefrontal-inhibition system was intact ${ }^{40,41}$. These differences suggest that the concept of excessive use of technology may be too broad. Instead, the excessive use of each technology can have its own etiology that should be studied independently.

It should also be noted that these findings can be inconsistent with some of the findings of two meta-analyses, which are also inconsistent in their results. In one meta-analysis of cuereactivity studies, people with IGD were found to exhibit no significant DLPFC impairment and no differences in the striatum ${ }^{42}$. In another ${ }^{43}$, nucleus accumbens responses were consistent with what we observed here. Still, there was also increased activity in the inferior frontal gyrus. Such differences may be an artifact of the differences between our cuereactivity task (video-based) and prior studies, the use of artificial cutoffs for IGD classification in prior studies, and/or our focus on LOL vs. a different range of games that other studies have focused on. Such differences ultimately allude to the need to further study the brain etiology of IGD under different conditions and with different tasks.

The third hypothesis was based on findings from studies of the insula ${ }^{44}$, and suggested that deprivation from video gaming will result in increased insular activation when gamers are exposed to videogame cues. As a center for processing somatic markers and developing interoceptive awareness of "needs" ${ }^{45}$, the insula mediates cue-induced cravings in substance use contexts, such as smoking ${ }^{46,47}$ and opioid use ${ }^{48}$. We found that the left insula is activated when video gamers are deprived of gaming and are exposed to video gaming cues. This result not only supports our hypothesis (at least in a lateralized fashion), but also points to an 
additional potential similarity between IGD and other addictive behaviors in terms of the role of the insula ${ }^{44}$, and to the potentially overlooked role of the insula in understating online gaming behavior. The observed insular activation in response to deprivation from video games is similar to insular responses to deprivation from substance use ${ }^{45}$.

The last hypothesis suggested that the observed insular activation in the deprivation condition is associated with increased reward system activity and decreased prefrontal activity. This hypothesis was based on evidence derived from other addictive behaviors ${ }^{19,45,49,50}$, such as smoking, where deprivation states exert an influence on the dual neural systems via the insula ${ }^{17}$. The hypothesis also implied that deprivation states and the insula play a similar role in IGD, as in other addictive behaviors ${ }^{14}$. Our results support this hypothesis. The PPI analysis showed that under deprivation (but not satiety) conditions, left insula activation was positively associated with left ventral striatum activation, and negatively associated with left DLPFC activation. This lends support to the theoretically assumed role of insular activity in sensitizing dopaminergic systems and reducing the ability of key prefrontal regions to respond to the stimuli ${ }^{14}$. It points to further similarity between neural models of substance use ${ }^{45}$, gambling ${ }^{49}$, food intake ${ }^{22}$, and IGD.

From a practical standpoint, our findings suggest that the insula can be a target for interventions developed in future research for treating IGD. Its activity can be modulated with transcranial direct current stimulation ${ }^{51}$ and repeated transcranial magnetic stimulation ${ }^{52}$. Disrupting its activity can extinguish addictive behaviors such as smoking ${ }^{45}$. Thus, future research can consider testing insula-based interventions for reducing IGD. Moreover, our findings can also explain changes in video gaming behavior in subjects with insular lesions ${ }^{53}$. Despite these promising findings and implications, our results should be interpreted with caution, given the limitations of our sample and design. Our sample was small, included 
mostly young-adult males, all from one country (China), all right-handed, and all played a specific video game. Generalizability to other populations and video games should be established in future research. These limitations may also underlie the inconsistent laterality we observed here: while most deprivation processes ( $\mathrm{H} 3$ and $\mathrm{H} 4)$ were lateralized to the left, IGD-related deficits (H2) were lateralized primarily to the right. This could be an artifact of the right-handedness of the sample, the fact that it is male-dominant, or its size, which may not have afforded to detect bilateral processes. Future research should examine such issues. In addition, we measured cue responses in an experimental setting, where the cues may not be as potent as real cues (e.g., watching one's own game). While the cues were sufficiently potent to elicit the expected activations, future research should consider different designs that include more naturalistic cues, and extend the cue-reactivity paradigm we employ here to capture behavioral measures (e.g., by using a go/no-go paradigm). It may also extend our findings by examining associations between craving and neural activity.

\section{Conclusion}

Video gaming can benefit many users. Nevertheless, it can result in some gamers in high levels of IGD. We sought to examine the neural underpinnings of IGD as a first step in developing interventions aimed at IGD preventions and reduction. Our findings lend empirical support to a tripartite model of IGD, according to which the interaction between reward, inhibition, and interoceptive awareness brain systems determines responses to video gaming cues, and is associated with IGD symptoms. 
Data availability: The data that support the findings of this study are available from the corresponding author upon reasonable request.

This article is protected by copyright. All rights reserved. 


\section{References:}

1. Blayzyte A. Gaming in China - Statistics and facts Statista. https://www.statista.com/topics/4642/gaming-in-china/. Published 2019. Accessed October 15, 2019.

2. Bavelier D, Green CS, Pouget A, Schrater P. Brain Plasticity Through the Life Span: Learning to Learn and Action Video Games. In: Hyman SE, ed. Annual Review of Neuroscience, Vol 35. Vol 35.2012:391-416.

3. Pujol J, Fenoll R, Forns J, et al. Video gaming in school children: How much is enough? Ann Neurol. 2016;80(3):424-433.

4. Turel O, Bechara A. Little video-gaming in adolescents can be protective, but too much is associated with increased substance use. Substance Use \& Misuse. 2019;forthcoming:1-11.

5. King DL, Haagsma MC, Delfabbro PH, Gradisar M, Griffiths MD. Toward a consensus definition of pathological video-gaming: A systematic review of psychometric assessment tools. Clinical psychology review. 2013;33(3):331-342.

6. Palaus M, Marron EM, Viejo-Sobera R, Redolar-Ripoll D. Neural Basis of Video Gaming: A Systematic Review. Front Hum Neurosci. 2017;11(248).

7. Kardefelt-Winther D, Heeren A, Schimmenti A, et al. How can we conceptualize behavioural addiction without pathologizing common behaviours? Addiction. 2017;112(10):1709-1715.

8. Billieux J, King DL, Higuchi S, et al. Functional impairment matters in the screening and diagnosis of gaming disorder: Commentary on: Scholars' open debate paper on the World Health Organization ICD-11 Gaming Disorder proposal (Aarseth et al.). $J$ Behav Addict. 2017;6(3):285-289.

9. American Psychiatric Association. Internet Gaming Disorder. In: Diagnostic and statistical manual of mental disorders (5th ed.). 5th ed. Arlington, VA: American Psychiatric Publishing; 2013:795-798.

10. World Health Organization. International classification of diseases for mortality and morbidity statistics (11th Revision). World Health Organization;2018.

11. Dong G, Potenza MN. A cognitive-behavioral model of Internet gaming disorder: Theoretical underpinnings and clinical implications. Journal of Psychiatric Research. 2014;58:7-11.

12. Bechara A. Decision-making, impulse control, and loss of willpower to resist drugs: A neurocognitive perspective. Nat Neurosci. 2005;8(11):1458-1463.

13. Bechara A, Berridge KC, Bickel WK, Morón JA, Williams SB, Stein JS. A Neurobehavioral Approach to Addiction: Implications for the Opioid Epidemic and the Psychology of Addiction. Psychological Science in the Public Interest. 2019;20(2):96-127.

14. Wei L, Zhang S, Turel O, Bechara A, He Q. A Tripartite Neurocognitive Model of Internet Gaming Disorder. Frontiers in Psychiatry. 2017;8(285):1-11.

15. McClure SM, Bickel WK. A dual-systems perspective on addiction: contributions from neuroimaging and cognitive training. Annals of the New York Academy of Sciences. 2014;1327(1):62-78.

16. Bechara A, Damasio H. Decision-making and addiction (part I): impaired activation of somatic states in substance dependent individuals when pondering decisions with negative future consequences. Neuropsychologia. 2002;40(10):1675-1689. 
17. Wood SMW, Bechara A. The neuroscience of dual (and triple) system in decision making. In: Reyna VF, Zayas V, eds. The Neuroscience of Risky Decision Making. Washington, DC: American Psychological Assoication; 2014:177-202.

18. Naqvi NH, Bechara A. The hidden island of addiction: The insula. Trends in Neurosciences. 2009;32(1):56-67.

19. Naqvi NH, Bechara A. The insula and drug addiction: an interoceptive view of pleasure, urges, and decision-making. Brain Structure \& Function. 2010;214(56):435-450.

20. Naqvi NH, Gaznick N, Tranel D, Bechara A. The insula: a critical neural substrate for craving and drug seeking under conflict and risk. In: Kingstone A, Miller MB, eds. Year in Cognitive Neuroscience. Vol 1316.2014:53-70.

21. Naqvi NH, Rudrauf D, Damasio H, Bechara A. Damage to the insula disrupts addiction to cigarette smoking. Science. 2007;315(5811):531-534.

22. He Q, Huang X, Zhang S, Turel O, Ma L, Bechara A. Dynamic Causal Modeling of Insular, Striatal, and Prefrontal Cortex Activities During a Food-Specific Go/NoGo Task. Biological Psychiatry: Cognitive Neuroscience and Neuroimaging. forthcoming.

23. He Q, Li D, Turel O, Bechara A, Hser Y-I. White matter integrity alternations associated with cocaine dependence and long-term abstinence: Preliminary findings. Behav Brain Res. 2020;379:112388.

24. Yuan K, Yu D, Cai C, et al. Frontostriatal circuits, resting state functional connectivity and cognitive control in internet gaming disorder. Addiction Biology. 2017;22(3):813-822.

25. Weinstein A, Livny A, Weizman A. New developments in brain research of internet and gaming disorder. Neuroscience \& Biobehavioral Reviews. 2017;75:314-330.

26. Ko C-H, Liu G-C, Hsiao S, et al. Brain activities associated with gaming urge of online gaming addiction. Journal of Psychiatric Research. 2009;43(7):739-747.

27. Lee J, Lee S, Chun JW, Cho H, Kim D-j, Jung Y-C. Compromised prefrontal cognitive control over emotional interference in adolescents with Internet Gaming Disorder. Cyberpsychology, Behavior, and Social Networking. 2015;18(11):661-668.

28. Kuss DJ, Griffiths MD, Pontes HM. Chaos and confusion in DSM-5 diagnosis of Internet Gaming Disorder: Issues, concerns, and recommendations for clarity in the field. J Behav Addict. 2017;6(2):103-109.

29. Koepp MJ, Gunn RN, Lawrence AD, et al. Evidence for striatal dopamine release during a video game. Nature. 1998;393(6682):266.

30. Montag C, Weber B, Trautner P, et al. Does excessive play of violent first-personshooter-video-games dampen brain activity in response to emotional stimuli? Biological Psychology. 2012;89(1):107-111.

31. Statista.com. Number of League of Legends monthly active users (MAU) from 2011 to 2016 (in millions). Statista. https://www.statista.com/statistics/317099/number-lolregistered-users-worldwide/. Published 2019. Accessed 2/5, 2019.

32. Carter BL, Tiffany ST. Meta-analysis of cue-reactivity in addiction research. Addiction. 1999;94(3):327-340.

33. Pontes HM, Griffiths MD. Measuring DSM-5 Internet gaming disorder: Development and validation of a short psychometric scale. ComputHum Behav. 2015;45:137-143.

34. Deleuze J, Long J, Liu T-Q, Maurage P, Billieux J. Passion or addiction? Correlates of healthy versus problematic use of videogames in a sample of French-speaking regular players. Addictive Behaviors. 2018;82:114-121. 
35. Brand M, Wegmann E, Stark R, et al. The Interaction of Person-Affect-CognitionExecution (I-PACE) model for addictive behaviors: Update, generalization to addictive behaviors beyond internet-use disorders, and specification of the process character of addictive behaviors. Neuroscience \& Biobehavioral Reviews. 2019;104:1-10.

36. Brand M, Young KS, Laier C, Wölfling K, Potenza MN. Integrating psychological and neurobiological considerations regarding the development and maintenance of specific Internet-use disorders: An Interaction of Person-Affect-Cognition-Execution (I-PACE) model. Neuroscience \& Biobehavioral Reviews. 2016;71:252-266.

37. Hofmann W, Friese M, Strack F. Impulse and Self-Control From a Dual-Systems Perspective. Perspectives on Psychological Science. 2009;4(2):162-176.

38. Jin CW, Zhang T, Cai CX, et al. Abnormal prefrontal cortex resting state functional connectivity and severity of internet gaming disorder. Brain Imaging and Behavior. 2016;10(3):719-729.

39. Kiraly O, Sleczka P, Pontes HM, Urban R, Griffiths MD, Demetrovics Z. Evaluation of the nine Internet Gaming Disorder criteria proposed in the DSM-5. J Behav Addict. 2016;5:23-23.

40. He Q, Turel O, Brevers D, Bechara A. Excess social media use in normal populations is associated with amygdala-striatal but not with prefrontal morphology. Psychiatry Research-Neuroimaging. 2017;269(1):31-35.

41. Turel O, He Q, Xue G, Xiao L, Bechara A. Examination of neural systems subserving Facebook "addiction". Psychol Rep. 2014;115(3):675-695.

42. Zheng H, Hu Y, Wang Z, Wang M, Du X, Dong G. Meta-analyses of the functional neural alterations in subjects with Internet gaming disorder: Similarities and differences across different paradigms. Progress in Neuro-Psychopharmacology and Biological Psychiatry. 2019;94:109656.

43. Starcke K, Antons S, Trotzke P, Brand M. Cue-reactivity in behavioral addictions: A meta-analysis and methodological considerations. J Behav Addict. 2018;7(2):227-238.

44. Abdolahi A, Williams GC, Benesch CG, et al. Damage to the insula leads to decreased nicotine withdrawal during abstinence. Addiction. 2015;110(12):1994-2003.

45. Droutman V, Read SJ, Bechara A. Revisiting the role of the insula in addiction. Trends in Cognitive Sciences. 2015;19(7):414-420.

46. Stippekohl B, Winkler M, Mucha RF, et al. Neural responses to BEGIN-and ENDstimuli of the smoking ritual in nonsmokers, nondeprived smokers, and deprived smokers. Neuropsychopharmacology. 2010;35(5):1209.

47. Moran-Santa Maria MM, Hartwell KJ, Hanlon CA, et al. Right anterior insula connectivity is important for cue-induced craving in nicotine-dependent smokers. Addiction biology. 2015;20(2):407-414.

48. Stewart JL, Khalsa SS, Kuplicki R, Puhl M, Investigators T, Paulus MP. Interoceptive attention in opioid and stimulant use disorder. Addiction Biology. forthcoming:e12831.

49. Clark L, Studer B, Bruss J, Tranel D, Bechara A. Damage to insula abolishes cognitive distortions during simulated gambling. Proceedings of the National Academy of Sciences of the United States of America. 2014;111(16):6098-6103.

50. Turel O, He Q, Brevers D, Bechara A. Delay discounting mediates the association between posterior insular cortex volume and social media addiction symptoms. Cognitive, Affective, \& Behavioral Neuroscience. 2018;18(4):694-704. 
51. Meng Z, Liu C, Yu C, Ma Y. Transcranial direct current stimulation of the frontalparietal-temporal area attenuates smoking behavior. Journal of Psychiatric Research. 2014;54:19-25.

52. Dinur-Klein L, Dannon P, Hadar A, et al. Smoking cessation induced by deep repetitive transcranial magnetic stimulation of the prefrontal and insular cortices: a prospective, randomized controlled trial. Biological psychiatry. 2014;76(9):742-749.

53. Cereda C, Ghika J, Maeder P, Bogousslavsky J. Strokes restricted to the insular cortex. Neurology. 2002;59(12):1950-1955. 


\section{Tables}

Table 1 Brain regions showing significant interaction between group and stimuli

\begin{tabular}{ccccccc}
\hline \multirow{2}{*}{ L/R } & \multirow{2}{*}{ Brain Region } & \multirow{2}{*}{ No of Voxels } & \multicolumn{4}{c}{ MNI Coordinates } \\
& & & $\mathrm{x}$ & $\mathrm{y}$ & $\mathrm{z}$ & $\mathrm{z}$ \\
\hline $\mathrm{L}$ & Frontal Pole & 479 & -26 & 36 & -4 & 5.04 \\
$\mathrm{~L} / \mathrm{R}$ & Ventral Striatum extending to & 395 & 6 & 28 & -14 & 4.26 \\
$\mathrm{R}$ & VMPFC & 185 & 46 & 14 & 22 & 4.84 \\
\hline
\end{tabular}

Table 2 Brain regions showing significant main effects of session (deprivation $>$ satiety)

\begin{tabular}{ccccccc}
\hline \multirow{2}{*}{ /R } & \multirow{2}{*}{ Brain Region } & \multirow{2}{*}{ No of Voxels } & \multicolumn{4}{c}{ MNI Coordinates } \\
& & & $\mathrm{X}$ & $\mathrm{y}$ & $\mathrm{z}$ & $\mathrm{z}$ \\
\hline $\mathrm{L}$ & Insula & 236 & -36 & 10 & -8 & 5.22 \\
$\mathrm{R}$ & Insula & 144 & 36 & 8 & -2 & 4.50 \\
\hline
\end{tabular}

Table 3 Brain regions showing significant interaction between session and stimuli

\begin{tabular}{ccccccc}
\hline \multirow{2}{*}{ L/R } & \multirow{2}{*}{ Brain Region } & \multirow{2}{*}{ No of Voxels } & \multicolumn{4}{c}{ MNI Coordinates } \\
& & & $\mathrm{x}$ & $\mathrm{y}$ & $\mathrm{z}$ & $\mathrm{z}$ \\
\hline $\mathrm{L}$ & Insula & 308 & -34 & 0 & 4 & 5.30 \\
$\mathrm{R}$ & DLPFC & 284 & 44 & 32 & -16 & 4.41 \\
$\mathrm{~L}$ & DLPFC & 206 & -42 & 32 & -16 & 4.34 \\
$\mathrm{R}$ & Ventral Striatum & 105 & 8 & 10 & -6 & 4.83 \\
\hline
\end{tabular}

Table 4 PPI results with the left insular cortex as seed region

\begin{tabular}{|c|c|c|c|c|c|c|}
\hline \multirow{2}{*}{$\mathrm{L} / \mathrm{R}$} & \multirow{2}{*}{ Brain Region } & \multirow{2}{*}{ No of Voxels } & \multicolumn{3}{|c|}{ MNI Coordinates } & \multirow{2}{*}{$\begin{array}{c}Z- \\
\text { score }\end{array}$} \\
\hline & & & $\mathrm{x}$ & $\mathrm{y}$ & $\mathrm{z}$ & \\
\hline \multicolumn{7}{|c|}{ Deprivation Condition (LOL > Control Videos) } \\
\hline $\mathrm{L}$ & Ventral Striatun & 151 & -24 & -6 & 8 & 4.28 \\
\hline \multicolumn{7}{|c|}{ Deprivation Condition (Control > LOL Videos) } \\
\hline $\mathrm{L}$ & DLPFC & 162 & -50 & 0 & 6 & 5.09 \\
\hline
\end{tabular}


NONE

Satiety Condition (LOL > Control Videos)

Satiety Condition (Control > LOL Videos)

NONE

This article is protected by copyright. All rights reserved. 


\section{Figures}

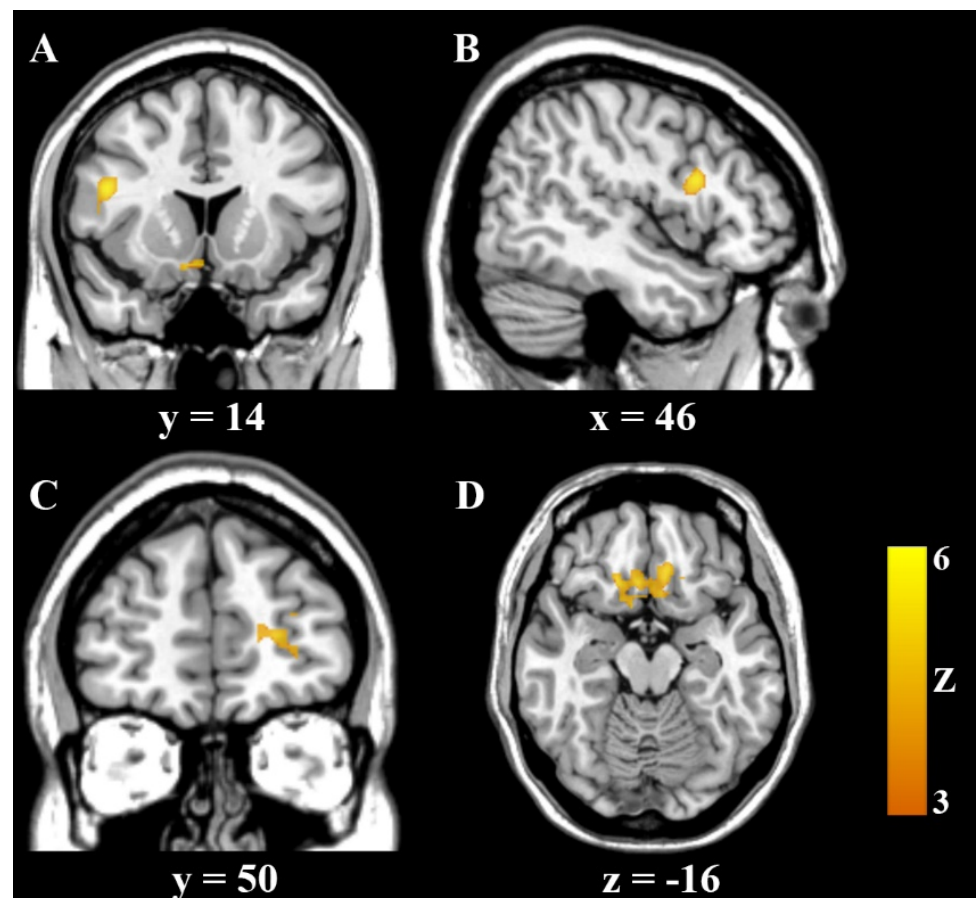

Figure 1: Brain regions that showed a significant interaction effect of stimuli by group. A) ventral striatum; B) right dorsolateral prefrontal cortex; C) left frontal pole; D) ventromedial prefrontal cortex. Images were mapped onto a standard brain with the left side of the paper represent the right side of the brain for A), C), and D). The numbers below each subplot indicate the slice number. The color bar represents the $\mathrm{Z}$ statistics. 
$\mathbf{A}_{50}$

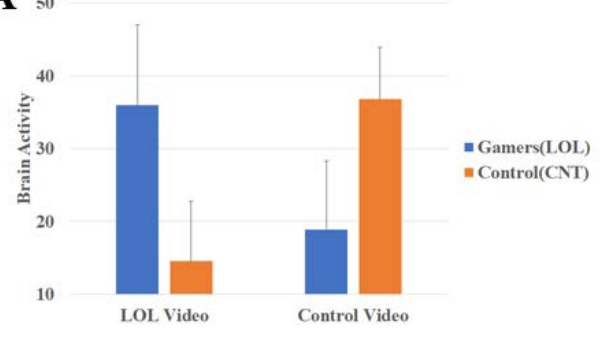

$\mathbf{C}^{15}$

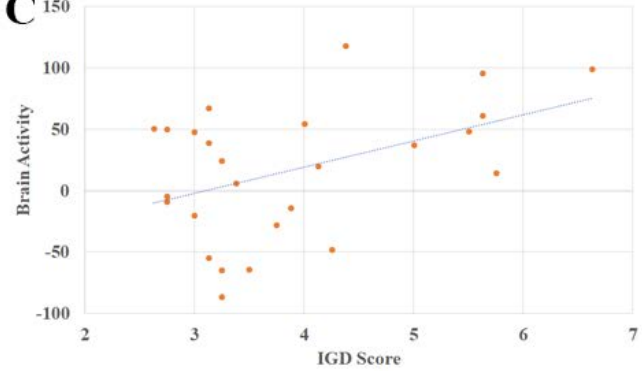

B

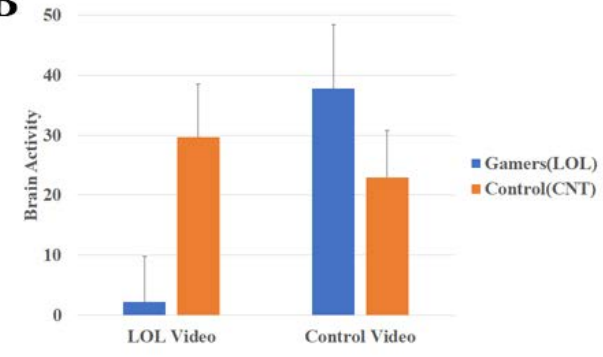

$\mathbf{D}^{1}$

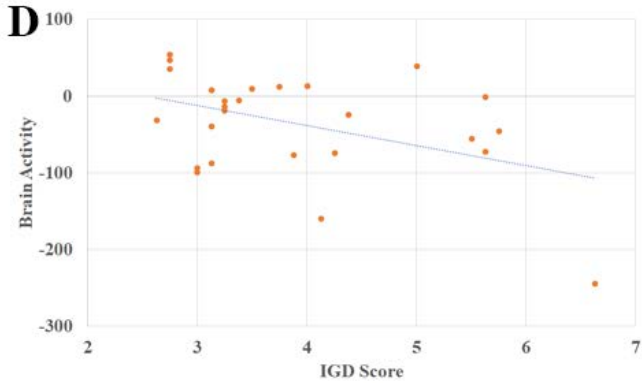

Figure 2: Brain activity pattern of the right ventral striatum (A) and right DLPFC showed an interaction between group and stimuli. Results suggested that the right striatum responded with higher activity when the LOL group watched LOL videos compared to when they watched control videos; and when the control group watched the control video compared to when they watched LOL videos. In contrast, when the LOL group watched LOL videos, they showed less prefrontal activity compared to when watching the control video. The control group did not show such differences. Brain behavior correlation also suggested that in the LOL group IGD scores were positively correlated with the activity difference of the right striatum when watching LOL videos vs. control videos (C), and negatively correlated with the activity difference of the right DLPFC when watching LOL videos vs. control videos (D). 


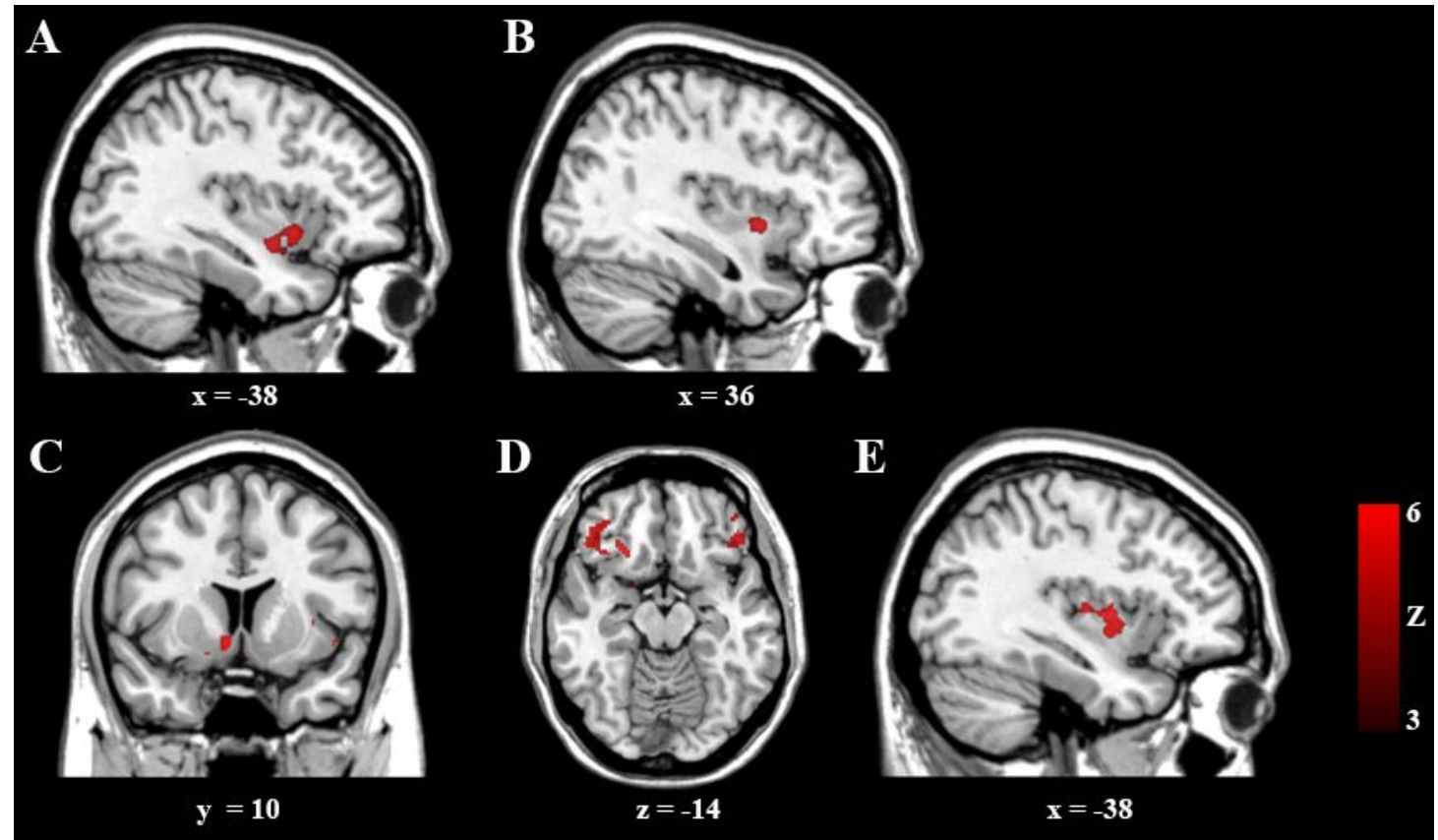

Figure 3: the left (A) and right (B) insular cortex showed a significant main effect of "session." There were significant interaction effects in the right ventral striatum (C), bilateral DLPFC region (D), as well as the left insular cortex (E). Images were mapped onto a standard brain with the left side of the paper representing the right side of the brain for (C) and (D). The numbers below each subplot indicate the slice number. Color bar represent the $\mathrm{Z}$ statistics. 

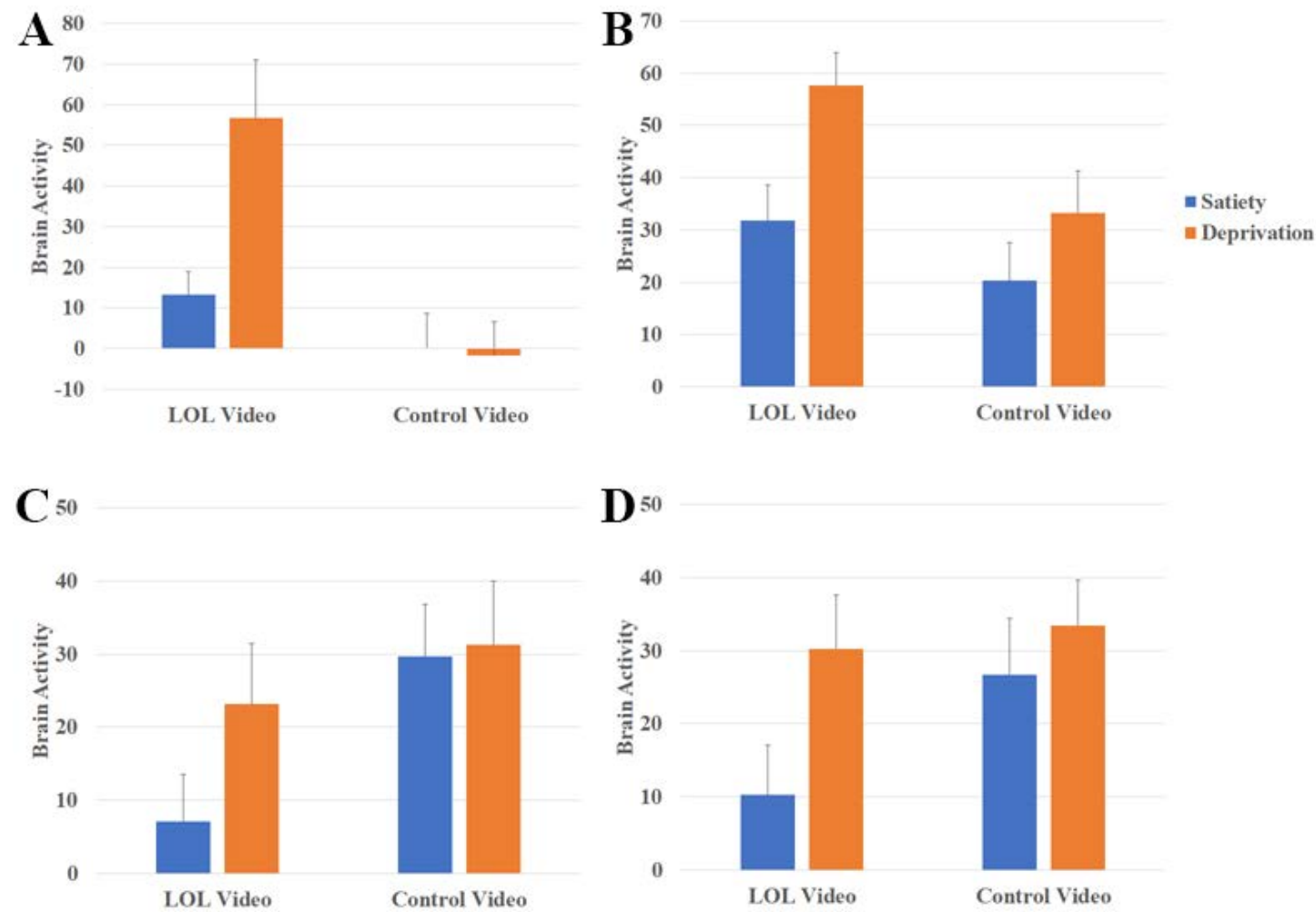

Figure 4: The activity of the left insular cortex (A), the right ventral striatum (B), the left (C), and right (D) DLPFC showed interaction of session and stimuli. Activity in the left insular cortex was higher when watching LOL videos in the deprivation condition than in all other conditions (A). Activity in the right striatum was higher in deprivation condition than in satiety condition when participants watched LOL video (B). Bilateral DLPFC activation was lower in the satiety condition than in the deprivation condition when watching LOL videos (C and D). Error bar represented the standard error. 


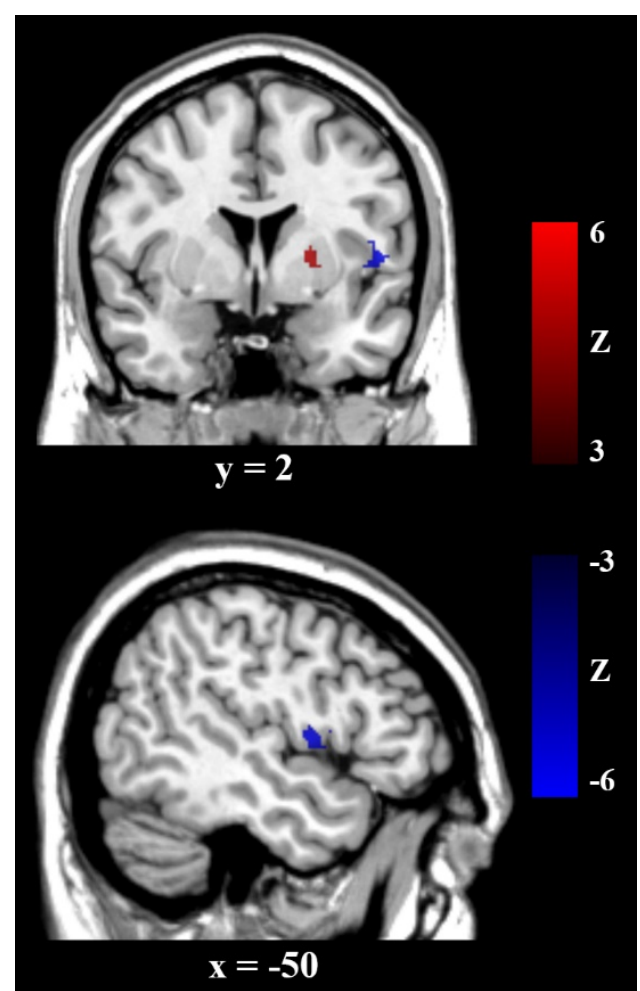

Figure 5: Using the left insular cortex as a region of interest, PPI analysis showed increased coupling between the left insula and ventral striatum (red region) when watching LOL videos compared to when watching control videos in the deprivation condition, but not in the satiety condition. It also showed decreased coupling between the left insula and left DLPFC (blue region) when watching LOL videos compared to when watching control videos in the deprivation condition, but not in the satiety condition.

Images were mapped onto a standard brain with the left side of the paper representing the right side of the brain for the upper panel. The numbers below each subplot indicate the slice number. Color bar represent the $\mathrm{Z}$ statistics. 


\title{
Supplementary Materials
}

\section{A. IGD Scale}

\author{
IGD Scale- Short Form (borrowed from Pontes, Griffiths ${ }^{33}$ ) \\ Reflecting on your LoL play in the latest 3-month period, to what extent.... \\ [1=very low extent/never; 7=very high extent/very frequently]
}

1. Do you feel preoccupied with your gaming behavior? (Some examples: Do you think about previous gaming activity or anticipate the next gaming session? Do you think gaming has become the dominant activity in your daily life?)

2. Do you feel more irritability, anxiety or even sadness when you try to either reduce or stop your gaming activity?

3. Do you feel the need to spend an increasing amount of time engaged in gaming in order to achieve satisfaction or pleasure?

4. Do you systematically fail when trying to control or cease your gaming activity?

5. Have you lost interest in previous hobbies and other entertainment activities because of your engagement with the game?

6. Have you continued your gaming activity despite knowing it was causing problems between you and other people?

7. Have you deceived any of your family members, therapists, or others because of the amount of your gaming activity?

8. Do you play in order to temporarily escape or relieve a negative mood (e.g., helplessness, guilt, anxiety)?

9. Have you jeopardized or lost an important relationship, job or an educational or career opportunity because of your gaming activity?

\section{B. Sample Stimuli}

External File A: Supplemental-LOL video. AVI

External File A: Supplemental-Control video.AVI

C. Table S1- Ratings of the materials (1-7 Likert Scale, $N=15)$

This article is protected by copyright. All rights reserved. 


\begin{tabular}{|c|c|c|c|}
\hline Dimension & LOL video & Control video & Statistics \\
\hline familiarity & $5.67 \pm 1.18$ & $5.87 \pm 0.83$ & $t(14)=-0.59, p=0.57$ \\
\hline vividness & $4.80 \pm 1.21$ & $4.67 \pm 1.45$ & $t(14)=0.29, p=0.78$ \\
\hline colorfulness & $5.80 \pm 1.15$ & $5.87 \pm 0.99$ & $t(14)=-0.16, p=0.88$ \\
\hline
\end{tabular}

\section{D. fMRI Protocol Details}

The MRI scans were performed in a 3T Siemens MAGNETOM Tim/Trio scanner at the Brain Imaging Center at a research University. Participants lay in the supine position on the scanner bed. Foam pads were used to minimize head motion. They were instructed to rest and avoid head movement during the structural scan. During the fMRI scan, the task was back-projected onto a screen through a mirror attached to the head coil. Stimulus presentation and timing of all stimuli and response events were operationalized with Eprime (version 2.0) on an IBM-compatible PC. The T1-weighted 3D-Magnetization Prepared RApid Gradient Echo (MPRAGE) sequence was used for covering the whole brain for registration purpose (TR (repetition time)/TE (echo time) $=2530 / 3.39 \mathrm{~ms}$, flip angel $=7^{\circ}$, matrix $=256 \times 256$, 196 sagittal slices, $1 \mathrm{~mm}$ isotropic resolution). Functional MRI images were acquired with blood oxygen level dependent (BOLD) scan using a zshim gradient echo EPI (echo planer imaging) sequence with PACE (prospective acquisition correction). This specific sequence is designed to reduce signal loss in the prefrontal and orbitofrontal areas. The PACE option can help reducing the impact of head motion during data acquisition. The scanning parameters were: TR/TE $=2000 / 25$ ms; flip angle $=90^{\circ} ; 64 \times 64$ matrix size with resolution $3 \times 3 \mathrm{~mm}^{2}$. Thirty-one $3.5-\mathrm{mm}$ axial slices were used to cover the whole cerebral cortex and most of the cerebellum with no gaps. The slices were tilted about $30^{\circ}$ clockwise along the AC-PC plane to obtain better signals in the orbitofrontal cortex.

This article is protected by copyright. All rights reserved. 


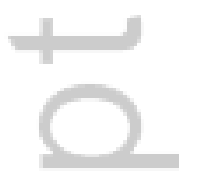

4 


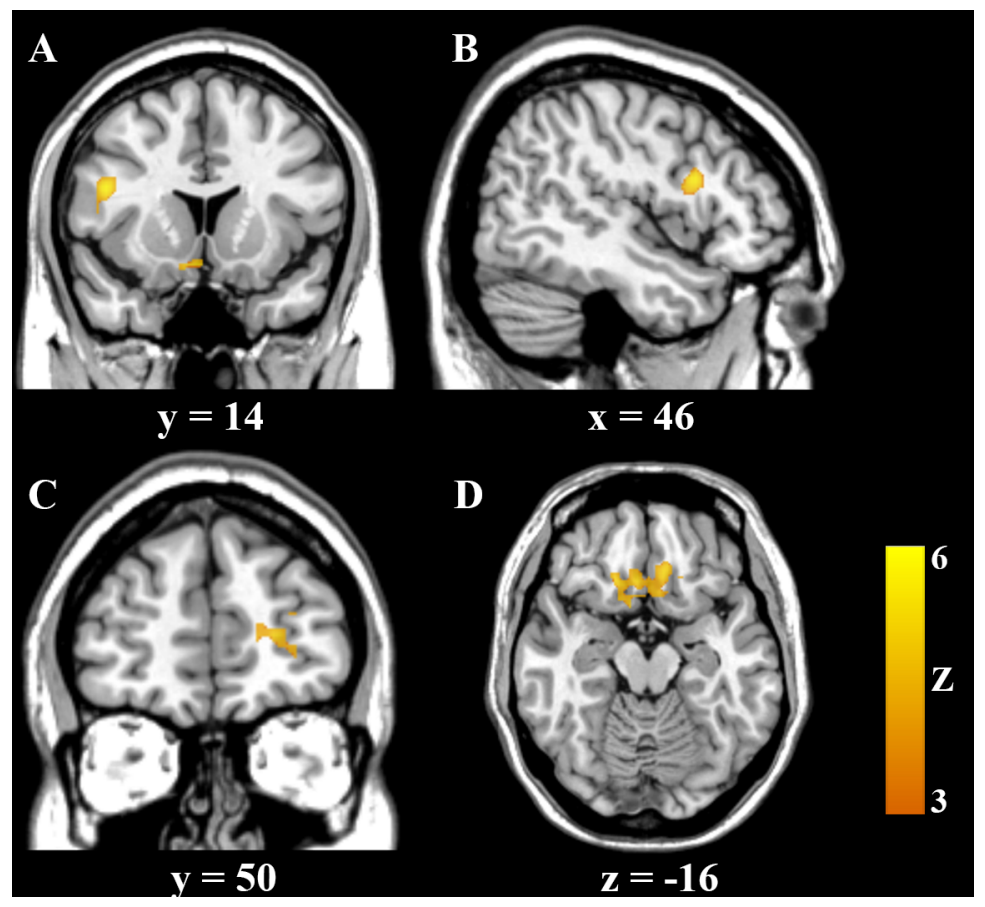

ADB_12894_fig1.tif

This article is protected by copyright. All rights reserved. 


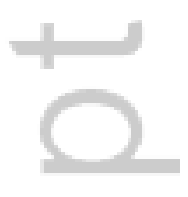

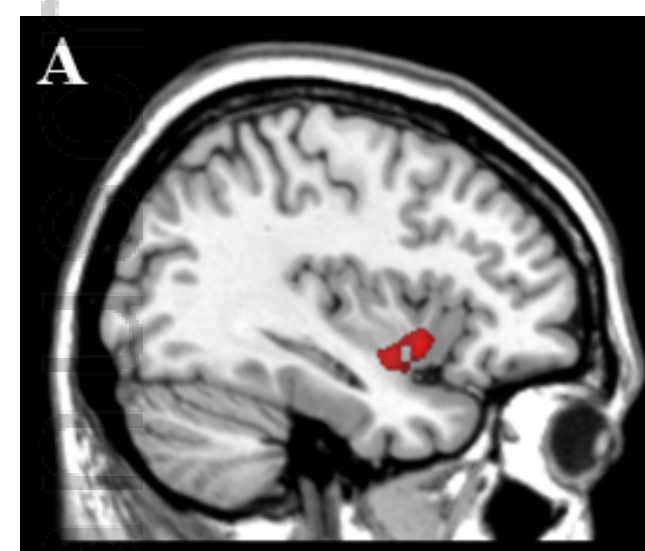

$$
x=-38
$$

\section{C}

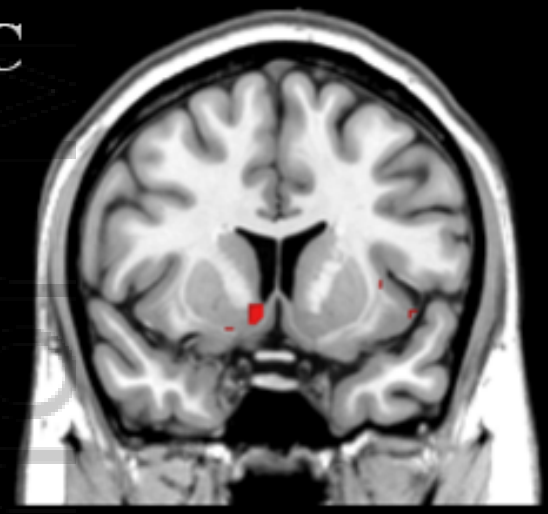

$$
y=10
$$

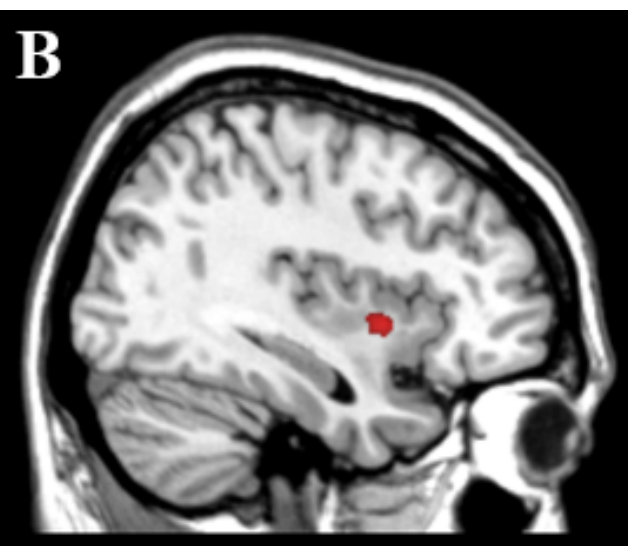

$x=36$

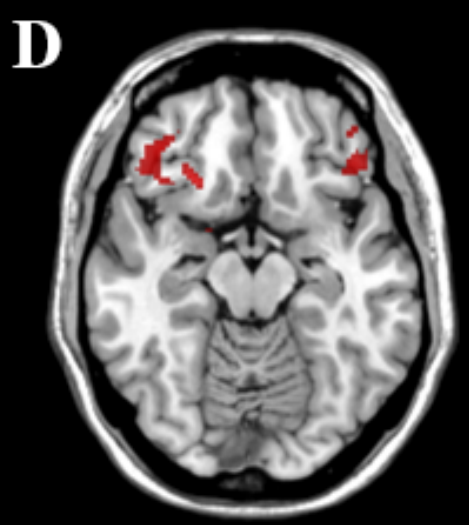

$z=-14$

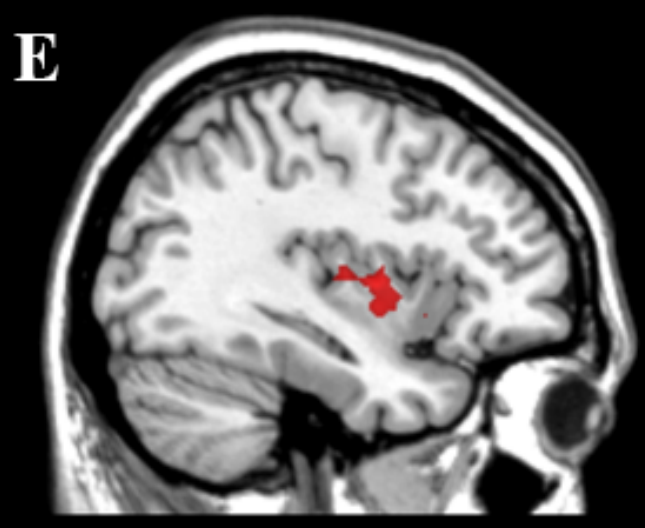

$\mathbf{x}=\mathbf{- 3 8}$

ADB_12894_fig3.tif 

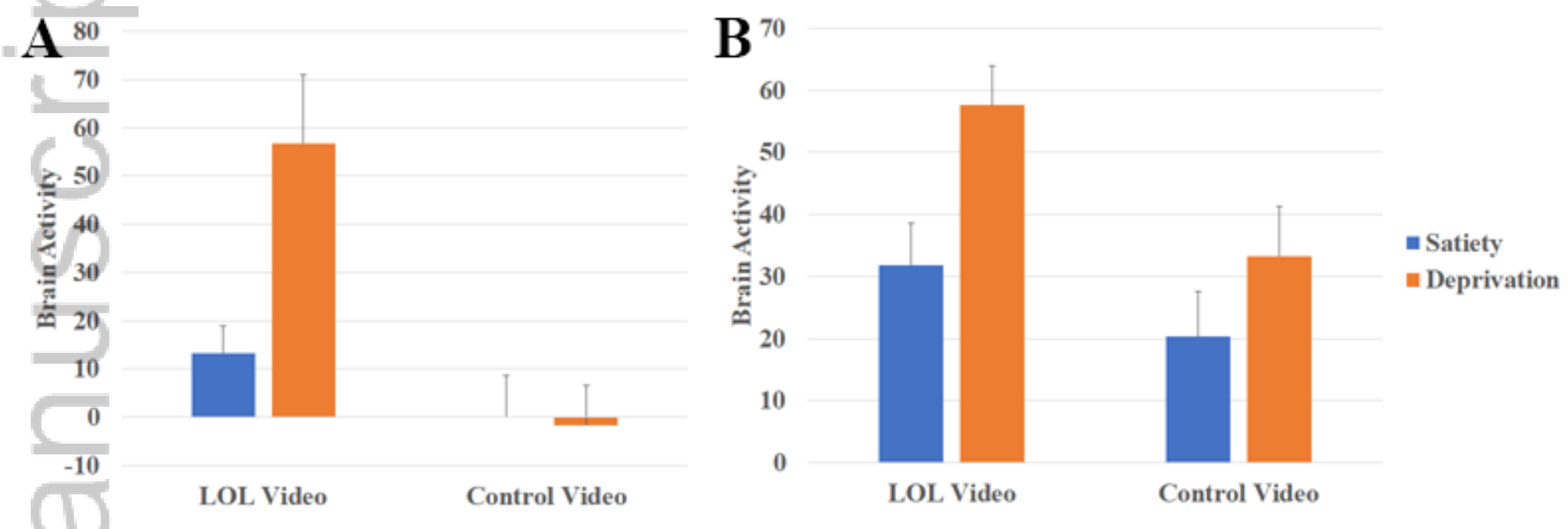

\section{$\mathrm{C}_{50}$}

\section{D $^{\text {50 }}$}
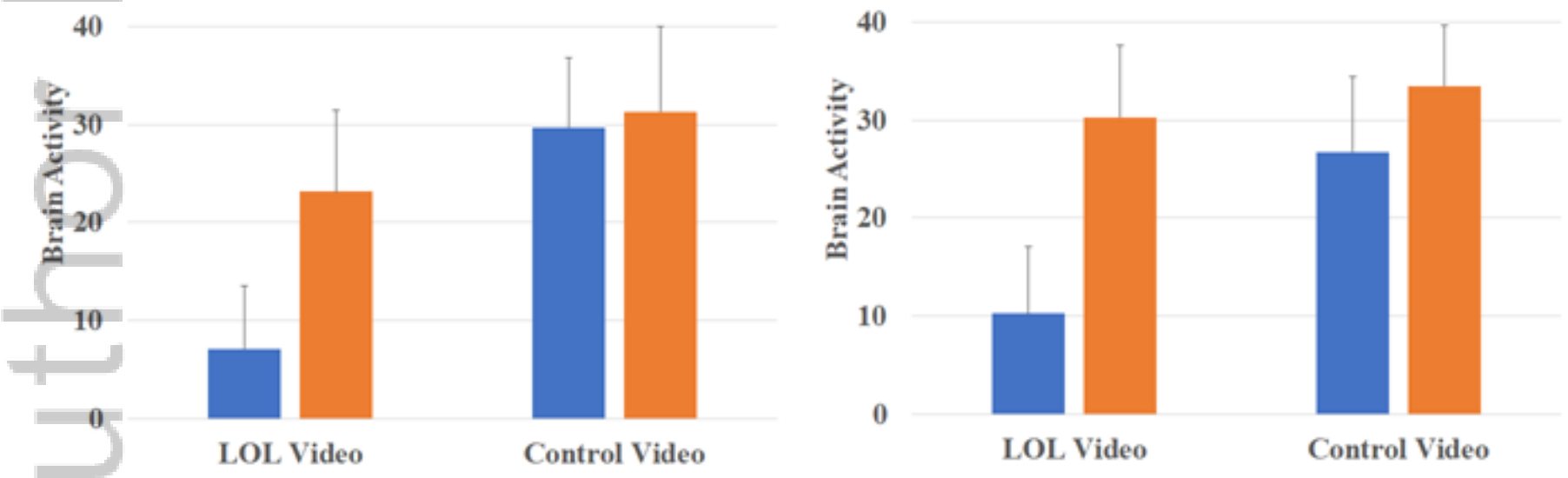

ADB_12894_fig4.tif

This article is protected by copyright. All rights reserved. 


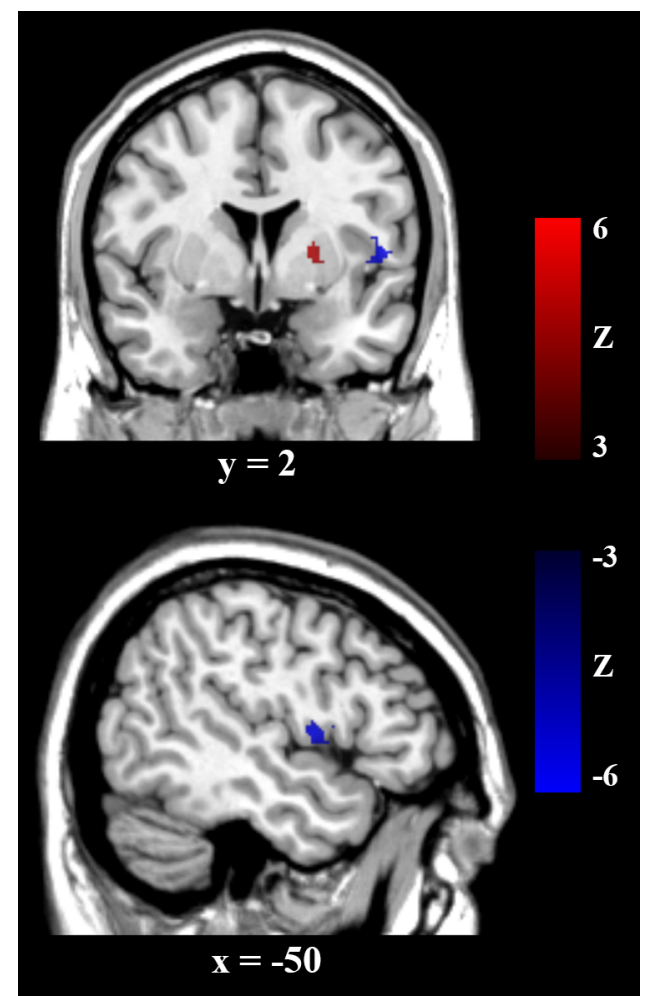

ADB_12894_fig5.tif

This article is protected by copyright. All rights reserved. 


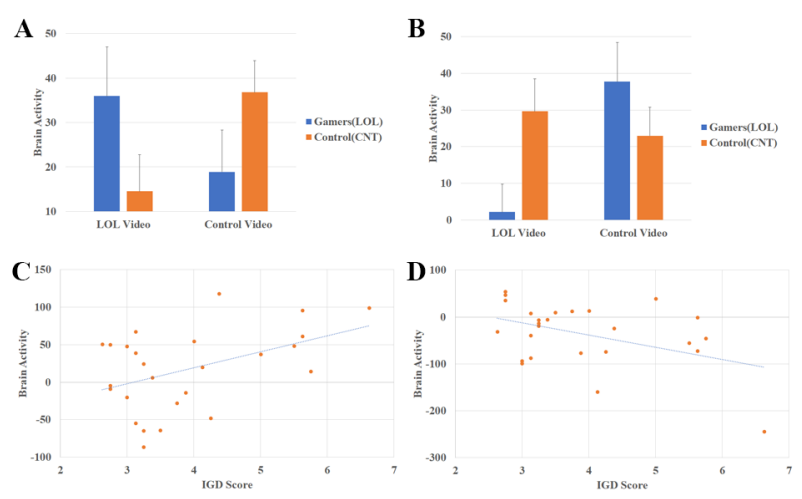

ADB_12894_Fig2.tif

This article is protected by copyright. All rights reserved. 


\section{University Library}

\section{- M M I E E R VA A gateway to Melbourne's research publications}

Minerva Access is the Institutional Repository of The University of Melbourne

Author/s:

Turel, O;He, Q;Wei, L;Bechara, A

Title:

The role of the insula in internet gaming disorder

Date:

2020-03-08

Citation:

Turel, O., He, Q., Wei, L. \& Bechara, A. (2020). The role of the insula in internet gaming disorder. ADDICTION BIOLOGY, 26 (2), https://doi.org/10.1111/adb.12894.

Persistent Link:

http://hdl.handle.net/11343/286965 